\title{
Design and research of underground parking intelligent monitoring system \\ Xinhong Yang
}

Shenyang Institute of Valuation Technoligy, Shengyang, 110045,China

Keywords: Monitor system, Underground parking lot, Construction system, Network

\begin{abstract}
Underground parking lot is a component of intelligent buildings, it has correlations with whole building as well as being a relatively dependent space. The safe reliability and stability of the parking lot monitor system is the core of the intelligent system. In this paper, through research and analysis, we use the network topology by combining the Client/Server and Browser/Server structure system. Using high-speed ball machine and gun type camera, network video server and central software or digital matrix, in order to ensure the complete function and reliable operation of the system. This system has strong resistance to interference and practicability and it can reduce construction cost and operation cost of the system.
\end{abstract}

\section{Introduction}

With development of the society and improvement of living standard, more and more people are purchasing automobiles for transportation. Automobile has become the major vehicle in modern society. According to the statistics, the volume of automobile ownership in China is nearly 140 million in 2014. In 2013, the number is 137 millions, which is 5.7 times of the number of automobile ownership back in 2003. Over the past decade, the annual growth of automobiles is 11 millions. Especially, the number of private car ownership exceeded 100 million in 2013 for the first time. Private cars make the transportation more free and comfortable. What ensued is to solve the parking problem. Many business districts, tourism areas, residential areas and so on set up parking lots. In recent years, many buildings are equipped with undergrounding parking lots in order to meet the actual demand of social life. At present, the management for parking lots varies regarding the quality. However, the management quality directly determines the passenger flow, occupancy rate and etc. This paper mainly conducts an analysis and study over the design of intelligent monitoring system of underground parking lots.

\section{Analysis of Current Situation}

As for the current underground parking lots, one way is manual that the monitoring is done by management staff of parking lots patrolling around, which needs the input of manpower and is not secure and reliable. Another way is taking the parking lots as a project in the design of intelligent building that is the common case for newly constructed high-rises over the past few years. Yet the monitoring system is dispersed and simplex. Therefore, the existing parking lots in operation often have an intelligent monitoring system with a integration mode combined with several independent systems. The integration, stability and expansibility of the system are unreliable. In addition, in the project of using the full bandwidth to solve the integration of multiple systems, most approaches are integrating the transmission of multimedia signal and controlling signal. Transmitting slow controlling signal in high-speed bandwidth is a waste of cyber resource. The practicality and operability is poor.

\section{The Design Concept of Monitoring System}

Based on the current situation of the management of underground parking lots, the design of monitoring system has high reliability and sustainability of development and can also reduce the construction cost of the system and the operation cost. The design of the system is reliable, stable, 
advanced and open. Meanwhile it is immune to interference, practical, expandable, universal and upgradable.

The monitoring system of parking lots is an essential facility in post-era of automobiles. While keeping the system up to date in the long run and maintaining the convenience of the interconnecting of systems and the general performance of ports, the system should adopt the advanced and open technology and products as much as possible.

Underground parking lot, as a part of the intelligent buildings, is related to the buildings complex, yet it is also an independent space. In design, it should be fully considered that the whole parking lot system is independent and stable, free from disturbance of each other. Thereby, it adopts the combination of network topology of the structure system of Client/Server and the structure system of Browser/Server. In the whole process of system design, it employs the speed dome, gun camera, network video server and central software (or digital matrix) to ensure the complete functions and reliable operation in line with the current application and future development.

The core performance of the parking lot monitoring system is the reliability and stability, which directly influences the application and practicability of the system. While designing, it must rule out the possibility that a single fault of one system could cause catastrophic loss for the application. Only this can ensure the basic application of the system. To adopt network topology of B/S system is mainly concerned with the safety and stability and to prevent the complete shut-down of the system due to a single malfunction at one part. If there is something wrong with single equipment or one line, it would not affect the whole system. In the meantime, the transmission of information data is carried out after encryption that the security and reliability are increased significantly.

In order to empower the monitoring system with strong vitality regarding application and lasting duration, it must be equipped with expandable and upgradable equipment and structure that is easy to be expanded and upgraded except for the above function. As for hardware, the video collection adopts the high-speed dome, gun camera, network video server, router and other equipments.

At the entrance and exit of the underground parking lot are both installed with a special gun camera of high definition for taking pictures of plate number; in the passages and parking spots, an infrared integrated camera with infrared light is installed at the height of 3 meters at every 50 meters. When the light is not good, it can automatically open the infrared light and maintain the monitoring function; at the four corner of the underground parking lot, a high-speed dome should be installed and can rotate to monitor the whole parking lot and send alarm.

The software utilizes the digital matrix. The network operating system of client selects multi-media access technology and system of multiple higher-level protocols. It adopts structured cabling at the client end, uses the LAN to transmit internal data, conducts long-range data transmission through linking internet with ADSL, increases the quantity of front-end video sources by upgrading, expanding, boarding, piling and etc. Meanwhile, network topology adopts B/S system. Users can increase or reduce video terminals at any time. System supports the video surveillance in real time and video access. The access of networking users does not affect the normal function of the system.

\section{Analysis and Study on the System}

The monitoring system of parking lots is made up of two major parts, internal monitoring and perimeter monitoring. The internal monitoring includes monitoring, data storage and inquiry, monitoring and alarming and security function (see illustration 1). The perimeter monitoring comprises monitoring and alarming. 


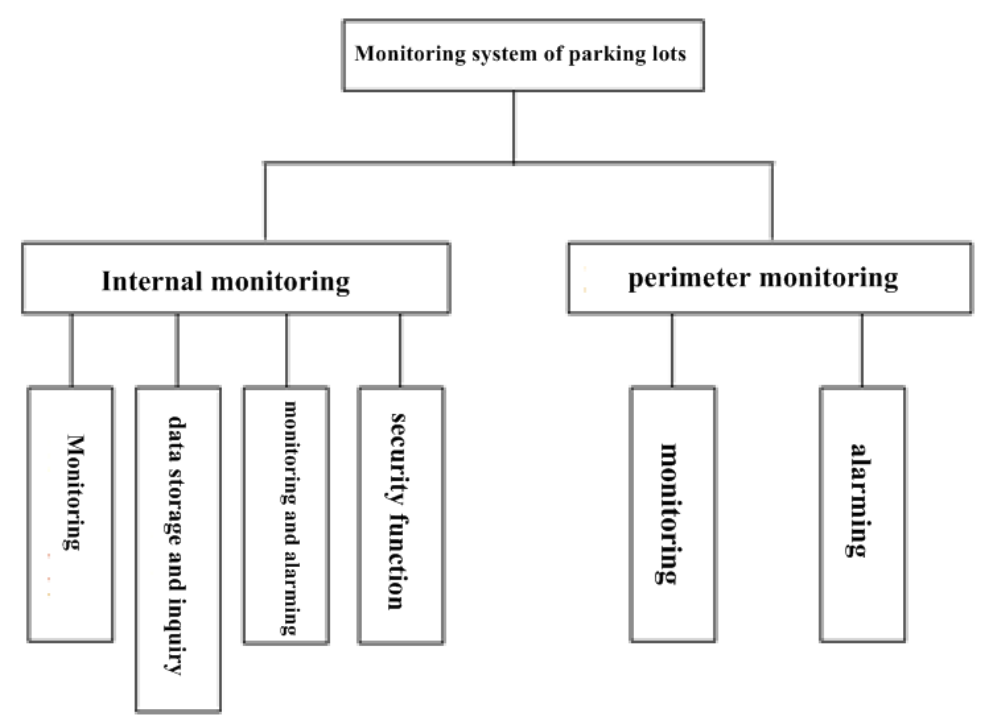

Fig. 1. Organization Chart of Monitoring System

\section{Internal Monitoring}

As the underground parking lot has a decentralized structure, the cabling is difficult and unsuitable. Therefore it is to use the WLAN as transmission mode which is easy for network construction, with short period, low investment and flexible structure. Using network video server covert the video signal collected by cameras into digital signal after compressing and then send the digital signal to monitoring center and transmit it to internet with ADSL. The administrators can open the client program in the computer connected with internet and input IP address of the corresponding video server and then can realize the overall remote monitoring and monitor the transmission of the monitoring video. Only realizing the visually monitoring is not enough, therefore there must also have storage and inquiry server in the parking lot so that monitoring can be comprehensive and run routing monitoring as well as storing the history data. The storage server can store the real-time audio and video and set a back-up. The inquiry server usually is set up in the monitoring room in parking lot. Through the server, the real situation of each monitoring point can be checked. If there is any abnormal situation, then it can send off alarm or make phone call to alarm.( see illustration 2)

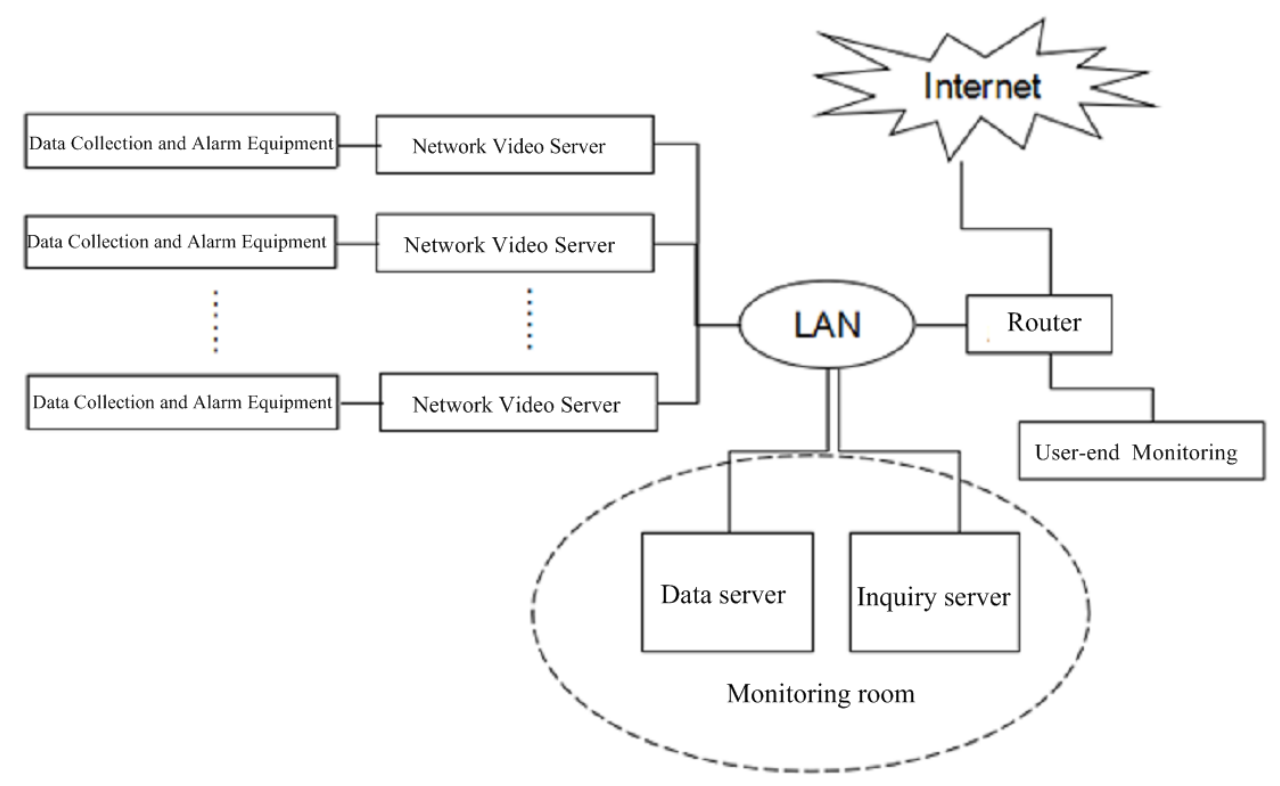

Fig. 2. The structure of parking lot monitoring system 


\section{Perimeter Monitoring}

Monitoring system should be set up also at the roads near the parking lot. This system is only to monitor the passing cars and passengers. The monitoring aim is very simple that is to prevent illegal actions of some people. When the monitoring captures movement of human face or loss of video signal in the targeted dynamic capturing area, it will send signal to the alarm in target area and automatically send off alarm to give a warning.

In order to make the monitoring more intuitive and clear, the monitoring center adopts digital matrix and can clearly monitor the parking lots and the traffic situation at the roads near it through converting the data into simulation signal and project it on TV with the help of network video decoder. When there is alarm at parking lots, digital matrix can automatically switch to video in that route and at the same time takes video and send the siren. With speaking on the microphone, the staff at the parking lot can be informed. Meanwhile, in order to realize the storage of large volume of video data, in addition to storage server, monitoring center cam configure RAID (Redundant Array of Inexpensive Disks) so as to meet the requirement of storing massive video as well as secure the video data.

\section{Conclusions}

Adopting advanced network technology and monitoring equipment to monitor parking lots and realize the intelligent and scientific management mode is the requirement of social progress. This paper studies the monitoring system of underground parking lots that can monitor the real situation and send off alarm and take action in case of abnormal situation. That is advantage of the monitoring system. However, car owners use mobile phones to conduct remote monitoring and the integration of the management system of IC intelligent card in parking lots and the system of network video monitoring need to be further studied. With putting in effort, reasonable utilization of modern technology and developing new project continually, it can create a safe and comfortable parking environment.

\section{References}

[1] Xiang He Qingxia Wu Experiment System of Remote Control Based on the C/S and B/S Mode . Electronics Optics and Control. 2005

[2] Congcong $\mathrm{Xu}$ Integrated Space Design of Urban Railway Stations and Surrounding Urban Complex. Paper Collection on Urban Development and Planning. 2011.

[3] Zhigang Ding Technology Research of Data Base Combined with C/S Mode and B/S Mode. Information Security and Technology. 2011.

[4] Yan Li Current Application Situation and Future Development Trend of Video Monitoring Technology, 2009 Annual Paper Collection of Information and Communication Network Technology Commission of China Institute of Communication. 2009.

[5] Tao Jia Deqiang Kong Yin Peng The Application of IP Multicast Technology in Video Monitoring Journal of Academic Communication of The 3rd National Signal and Intelligent Information Process and Application. 2009.

[6] Xudong Wu Yinli Application of Security Access System of Video Monitoring. Paper Collection of National Computer Security Academic Seminar. 2010. 